\title{
Centro de difusión de las humanidades (CDIHUM) en su primer aniversario de apertura
}

Alba Rodríguez Herrera

El pasado marzo del 2016 se cumplió el primer aniversario de la apertura del Centro de Difusión de las Humanidades (CDIHUM), de la Facultad de Humanidades y Ciencias Jurídicas de la UNAN-Managua. Es un centro de desarrollo integral, de usos múltiples, donde se le da cabida a la promoción de las publicaciones periódicas, bibliográfica, y a las actividades académicas integrales, entre ellas la investigación.

El CDIHUM nace bajo la iniciativa del Decano Doctor Luis Alfredo Lobato Blanco de aglutinar en un único espacio que prestara todas las condiciones, la información en físico y digital relacionada con las carreras que ofrece la Facultad. En ese sentido, las colecciones de los diferentes Centros de Documentación (CDOCS) de los Departamentos pasaron a formar parte del nuevo Centro.

Otra de los propósitos de la fundación del Centro, consiste en la creación y fortalecimiento de unidades especializadas en procesos de digitalización de documentos y manejo y uso de las TICs, con el fin de apuntalar hacia la convergencia del uso de las nuevas herramientas tecnológicas y los procesos de enseñanza-aprendizaje. Iniciativa que va caminando con buen suceso, y constituye una de las basas de la Facultad para el impulso de la educación virtual, a la que apuestan las autoridades de la Universidad,.

El Centro de Difusión de la Facultad de Humanidades y Ciencias Jurídicas de la UNAN-Managua, tiene como misión la de ser una unidad de información especializada en las áreas humanísticas, sociales y jurídicas. El objetivo es ofrecer servicios de información de calidad, oportuna y pertinente, apoyado por un equipo humano profesional comprometido en garantizar los servicios de información, los recursos bibliográficos y documentales. De igual manera, busca difundir el patrimonio intelectual producido por la Facultad, garantizando su conservación, preservación, acceso y difusión, siendo un valioso apoyo al aprendizaje, la docencia, la investigación y la gestión cultural.

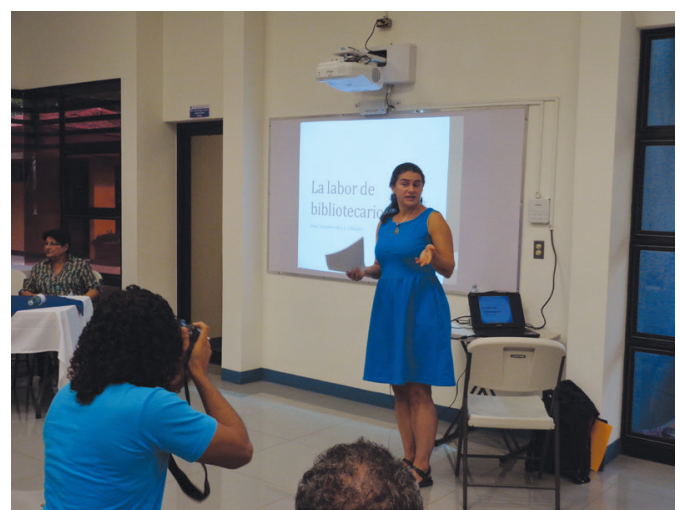

Melanie Polutta conferencista norteamericana invitada con estudiantes de la carrera Ciencias de la Información Fuente : Elvin Hernández, fecha 26 de agosto de 2015.
Autoridades presiden acto de inauguración del Centro de difusión de las humanidades (CDIHUM) 16 de marzo de 2016. Foto Alba Rodríguez. 


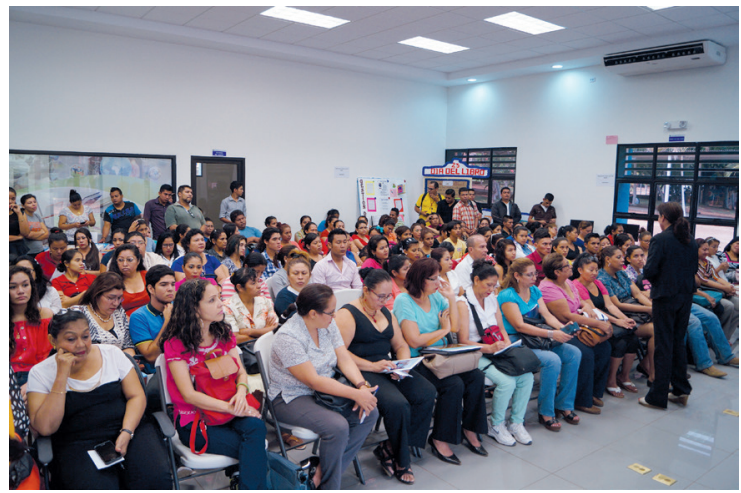

Actividad en ocasión al día del libro. Fecha: 23 de abril 2016. Fuente: Elvin Hernández

La visión estratégica que el Centro tiene, se inscribe dentro de las perspectivas que tiene la UNAN-Managua: ser la principal unidad de información en el campo humanístico, social y jurídico, reconocida por los servicios que presta en el ámbito académico, tanto en el nivel de grado como de postgrado. Con ello se pretende convertir en una instancia impulsadora de cambios con sentido científico y compromiso social, defensora del legado cultural e histórico del país y comprometido con el cuido del medio ambiente y la madre tierra.

La apertura del Centro, implicó un proceso que transitó desde la planificación y definición del proyecto, infraestructura y equipamiento, hasta la integración total de los fondos, la carta de servicios, y la contratación de personal para atender las tareas propias de los procesos. Los encargados de esta actividad fueron apoyados por el voluntariado de los alumnos ayudantes de la carrera de Gestión de la información y del departamento de Psicología. Durante el proceso de organización de las colecciones, se ha contado con la colaboración de los alumnos de III, IV y V año de la carrera de Gestión de la Información, en el marco de sus prácticas profesionales.

El Centro cuenta con una sala de lectura para atender a 50 usuarios, el área de análisis y

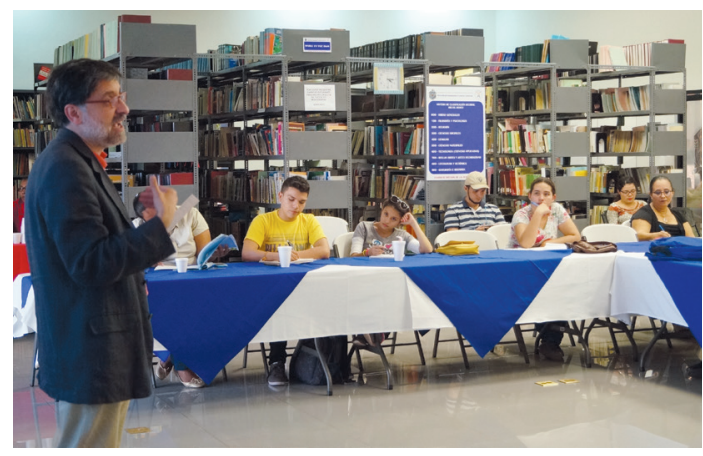

Doctor Ariel Arnal especialista del SIMO (Servicio de Inteligencia en Mercado y Opinión) y docente de la Universidad Autónoma de la Ciudad de México, imparte el taller "Cine documental e investigación disruptiva para el cambio social". Febrero 2016. Fuente: Martha Ortiz.

procesamiento documental y digital, Área de uso de las Tecnologías de Información (TIC), Edición de Revista Humanismo y Cambio Social y Área de Comunicación y divulgación. El fondo bibliográfico lo componen 12,000 libros entre títulos y copias, además se cuenta con 30 títulos de revistas nacionales e internacionales. De igual manera, se cuenta con 1,600 títulos de la colección monográfica, seminarios de graduación, maestrías y doctorados. Se cuenta con un horario de atención establecido, de08:00 a.m. a 4:30 p.m., de lunes a viernes.

Los sistemas de almacenamiento y recuperación de la información utilizados en el centro son: Sistema de Clasificación Decimal de Melvil Dewey, $2^{\text {a }}$ ed., Lista de Encabezamientos de materia para bibliotecas, Tabla de Cutter Sanborn, Sistema de Clasificación de Medicina de la biblioteca del Congreso de los Estados Unidos. Para la difusión y recuperación de la información, el sistema ABCD-ISIS, programa utilizado en todo el sistema bibliotecario de la UNANManagua.

Este proceso conlleva muchas actividades como el procesamiento de la información y la conformación de la base de datos bibliográficos referenciales: control de calidad de los registros, así como el proceso mecánico de los libros. 
A nivel de procesamiento, se han catalogado, clasificado e indizado 2, 689 títulos, además de registrado 4,913 libros. Como un dato a destacar está la atención de 1,689 alumnos de las diferentes carreras, quienes han consultado 1,684 libros de texto, 1,011 monografías, 38 obras de referencia (entre diccionarios, atlas, memorias, manuales y colecciones especiales), 56 libros especializados en el área médica.

La atención que da el Centro, va más allá de la población estudiantil y académica de la Facultad. En este sentido, se han atendido a 120 alumnos pertenecientes a otras facultades de la Universidad, que cursan asignaturas básicas del plan de estudios generales.

El centro cuenta con conexión Wifi permitiendo a los usuarios contar con servicio de Internet. Además, se cuenta con tres computadoras para uso de búsqueda en base de datos bibliográficos del centro, catálogos del sistema bibliotecario universitario, bases de datos por suscripción y otros.

En relación a la estructura organizativa del CDIHUM se puede resumir en las siguientes área: - Coordinación del CDIHUM a cargo de un docente de medio tiempo, especialista en

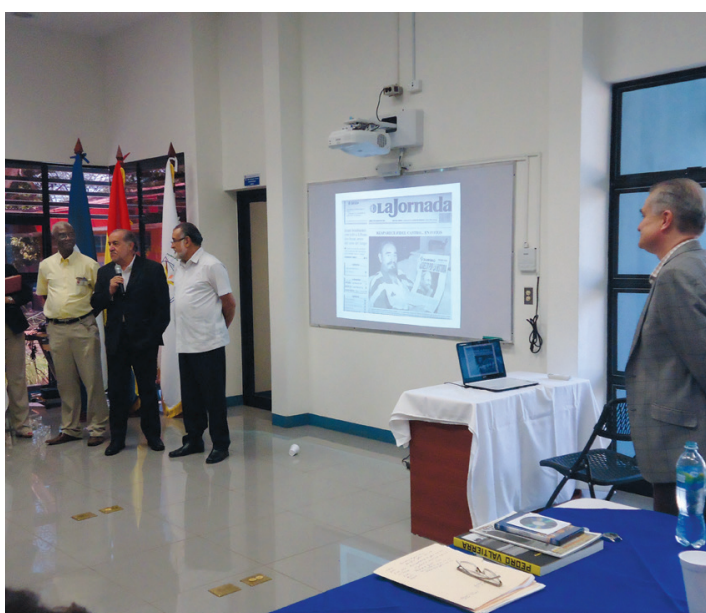

Doctor Alberto del Castillo Troncoso, académico del Instituto Mora de México impartiendo el taller "La fotografía como documento para la investigación". Febrero 2016.

Fuente: Departamento de Historia. gestión de la información. Se cuenta con una sala de lectura, atendida por dos asistentes de tiempo completo. El Área de Tecnología de la información y la comunicación (En esta área se encuentran: Soporte técnico, Área de análisis documental y Divulgación) atendida por especialista en TIC, diseño y comunicación social. El área de la edición de la Revista Humanismo y Cambio Social. Todo el centro es atendido por la Decana de Facultad con quien se tiene una comunicación fluida acerca de las actividades que se realizan.

Entre las perspectivas para seguir mejorando en el 2016 y los años subsiguientes están: la conformación del repositorio de la Facultad para integrarlo al repositorio institucional, así como continuar con el análisis documental y procesamiento técnico de las colecciones (1, 500 títulos y 1,000 copias). De igual manera, la digitalización de la colección monográfica, algunos libros históricos de ediciones agotadas (que están en formato papel), creación de nuevos servicios de información, referidos a la capacitación en el uso y manejo de bases de datos por suscripción y el digesto jurídico.

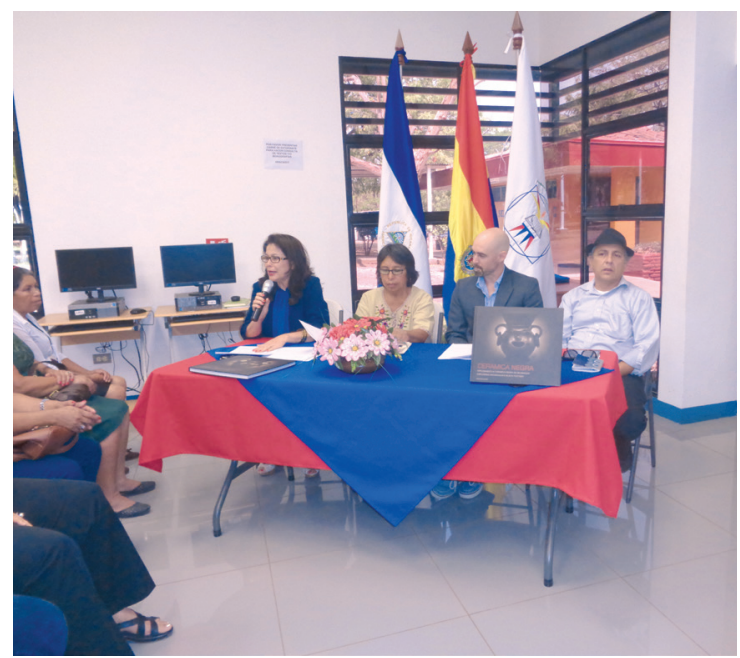

Presentación del libro Cerámica negra del fotógrafo norteamericano, Bryson Adams. Abril 2016. / Fuente : Alba Rodríguez 